\title{
Issues surrounding the cognitive neuroscience of obsessive-compulsive disorder
}

\author{
KEVIN D. WILSON \\ University of Pennsylvania, Philadelphia, Pennsylvania
}

\begin{abstract}
Obsessive-compulsive disorder (OCD) has been studied extensively in recent years, with increased emphasis on understanding OCD's biological substrates. There has been significant progress in documenting abnormal brain function in OCD patients, particularly in the orbitofrontal cortex, basal ganglia, and thalamus. Similar progress has broadened our understanding of the cognitive and behavioral manifestations of the disorder, including deficits in set shifting, hyperattention, and visuospatial construction abilities. Unfortunately, these results have not been replicated consistently. This report comprises a review of previous attempts to characterize the neurobiology and neuropsychology of OCD, and a discussion of several factors in OCD research that can help to explain previous inconsistencies.
\end{abstract}

Obsessive-compulsive disorder (OCD) is a relatively common form of psychopathology with a lifetime prevalence of $2 \%-3 \%$ (Robins et al., 1984). Although the anatomical and behavioral regularities of the disorder have been studied extensively over the past 20 years, there has been relatively little success in the attempt to establish how the former give rise to the latter. Popular models of OCD have drawn upon a variety of disciplines, including learning theory (Mowrer, 1960), cybernetics (Pitman, 1987) and psychoanalysis (Fenichel, 1945). Often, however, these models have had relatively little to say about the dysfunctional aspects of the OCD brain. This is due in part to the fact that, until recently, the neuroanatomical substrates of $O C D$ were poorly understood. Although $O C D$ researchers had long suspected the involvement of the frontal cortex and basal ganglia in OCD neuropathology, there was little empirical evidence to support such claims. Only through the use of modern neuroimaging techniques have many of these suspicions been confirmed.

Neurobiological studies of OCD have produced a wealth of data, but the inability to consistently replicate results has left many questions unanswered. Neuropsychological studies have similarly generated much information, but with equal uncertainty. An adequate theory of OCD must integrate the findings from each of these two disciplines, but any such integration would be premature at the moment, given the variability present within each. The goal of this review is therefore not to provide an all-encompassing theory of OCD expression, but rather to delineate several factors that may explain the discrepancies among previous studies of OCD. Consideration of these factors will help clarify the relevant neurobiological and neuropsycho-

This research was funded by NINDS Grant R01 NS34030 (issued to Martha J. Farah) and an NSF STC grant to the Institute for Research in Cognitive Science at the University of Pennsylvania. Correspondence concerning this manuscript should be addressed to K. D. Wilson, Department of Psychology, University of Pennsylvania, 3815 Walnut Street, Philadelphia, PA 19104 (e-mail: kwilson@psych.upenn.edu). logical features of OCD. Only then will an adequate theory of the cognitive neuroscience of OCD be possible.

\section{PHENOMENOLOGY OF OCD}

OCD is grouped under the general heading of anxiety disorders. The DSM-IV (American Psychological Association, 1994) defines OCD as "recurrent obsessions or compulsions that are severe enough to be time consuming (i.e., they take more than 1 hour a day) or cause marked distress or significant impairment." It defines obsessions as "persistent ideas, thoughts, impulses, or images that are experienced as intrusive and inappropriate and that cause marked anxiety or distress." It defines compulsions as "repetitive behaviors ... or mental acts ... the goal of which is to prevent or reduce anxiety or distress, not to provide pleasure or gratification." Unfortunately, these behaviors are performed with excessive frequency, or with little direct connection to the original purpose of the action.

OCD is differentiable from obsessive-compulsive personality disorder (OCPD) by feelings of inappropriateness with respect to the obsessive and compulsive behaviors. Whereas OCD patients find these thoughts and behaviors to be intrusive and inconsistent with their values and belief systems (ego-dystonic), OCPD patients maintain that these thoughts and actions are consistent with their self-image (ego-syntonic) (Hembree, Foa, \& Kozak, 1994). Although as many as $50 \%$ of OCD patients meet the criteria for OCPD (Rasmussen \& Tsuang, 1986), the two remain quite distinct in etiology, maintenance, and treatment.

One of the most striking features of the disorder is the degree of regularity in obsessional thoughts and compulsive behaviors. According to the $D S M-I V$, the most common obsessions are fear of contamination, thoughts of aggression, pathological doubt, excessive focus on bodily functions, need for order, and sexual imagery. The manual reports a similar regularity in compulsive behaviors, the most common being washing and cleaning, counting, 
checking, requesting or demanding assurances, repetition of previously performed actions, and ordering. Although some patients experience a single thought obsessionally, a majority experience more than one obsession or compulsion either simultaneously or over the course of illness (Rasmussen \& Tsuang, 1986).

Initial presentation of OCD typically occurs in adolescence or in the early twenties (Black, 1974), with the average onset occurring between ages 6 and 15 years for males and between ages 20 and 29 years for females. There is evidence for a genetic component, with concordance rates considerably higher for monozygotic twins than for dyzygotic twins (Stanley \& Prather, 1993). Similarly, first-degree relatives of OCD patients are at higher risk for psychopathology, with $25 \%$ being themselves diagnosed with OCD (Maxmen \& Ward, 1995).

It is estimated that as many as $80 \%$ of OCD patients suffer from depression (Rasmussen \& Tsaung, 1986), making it the most common co-occurring psychopathology in OCD (Barlow, 1988). Tic disorders are also common: Tourette's syndrome is evident in $10 \%-15 \%$ of OCD patients (Pauls, Leckman, Towbin, Zahner, \& Cohen, 1986). Up to $13 \%$ of female OCD patients are diagnosed with eating disorders (including anorexia and bulimia nervousa) (Maxmen \& Ward, 1995). Furthermore, anxiety disorders (e.g., panic disorder), delusional disorders, hypochondriasis, and personality disorders (e.g., histrionic) (Hembree et al., 1994), as well as Huntington's disease, Sydenham's chorea, and postencephalitic Parkinsonism (Cummings, 1993) have all been documented in cases of OCD. The overlap between schizophrenia and OCD appears to be relatively small, with less than $3 \%$ of OCD patients being diagnosed as schizophrenic (Black, 1974).

Several treatment options are currently available for OCD. Rational-emotive (Warren \& Zgourides, 1991), psychoanalytic (Oppenheim \& Rosenberger, 1991), and cognitive therapies (Salkovskis \& Warwick, 1985) have all been relatively unsuccessful. For example, Turner and Michelson (1984) estimate that $20 \%-40 \%$ of OCD patients show improvement with psychotherapy. Behavioral interventions, including exposure therapy and desensitization, have been more successful in clinical settings (Williams \& Chambless, 1994), with reviews estimating that $60 \%-80 \%$ of OCD patients improve with behavioral interventions (Turner \& Michelson, 1984).

Pharmacological treatments of OCD have become widely available in recent years. Selective serotonin reuptake inhibitors (SSRIs) such as clomipramine (Anaflaxine), fluoxetine (Prozac), and flouvoxamine (Floxyfral) have been the most successful agents in alleviating OCD symptoms (Perse, Greist, Jefferson, Rosenfeld, \& Dar, 1987). Response rates, however, are far from ideal. For example, approximately $70 \%$ of OCD patients respond to clomipramine treatment, with typical patients seeing only a $40 \%$ reduction in symptoms (Turner \& Michelson, 1984). Relapse occurs in approximately $85 \%$ of patients after termination of treatment.
Severe cases of OCD can be treated surgically. Several procedures, including thermocapsulotomy, cingulotomy, subcaudate tractatomy, and limbic leukotomy (Jenike et al., 1991) have all been documented as effective in relieving OCD symptoms, but each presents a host of potential complications. Although quite effective in most cases, the potential negative side effects justify their use only in the most intractable and debilitating cases.

\section{NEUROIMAGING OF OCD}

Recent advances in neuroimaging techniques have led to a number of significant findings in the $O C D$ literature (for a more complete review, see Trivedi, 1996). In several studies, researchers have looked for structural abnormalities that differentiate OCD patients and controls by using methods such as MRI and CT. In other studies, researchers have looked for differences in regional brain activity during symptom provocation or in association with particular treatments, using methods such as PET, SPECT, and $f$ MRI. Although a number of conflicting results will be reviewed here, the majority of studies suggest neuropathology in three regions: the orbitofrontal cortex, the basal ganglia (specifically, the head of the caudate nucleus), and the thalamus.

\section{Structural Brain Imaging Studies}

Insel, Donnelly, Lalakea, Alterman, and Murphy (1983), using CT, conducted one of the earliest neuroimaging studies of OCD. These authors compared 10 OCD patients and 10 healthy controls on a number of brain measures, including ventricular size and hemispheric asymmetry, and found no significant differences between groups. Behar et al. (1984) conducted a similar CT study, using 16 adolescent $O C D$ patients, but reported ventricular enlargement in the patient group relative to controls. This finding was not replicated, however, by Luxenberg et al. (1988), who found no significant difference in ventricular size between OCD and control subjects $(n=10$ in each group), although these authors did note a nonsignificant trend in that direction. Nevertheless, Luxenberg et al. did find smaller caudate nuclei in the OCD patient group relative to controls. Kellner et al. (1991), in contrast, reported no difference between $O C D$ patient and control subjects on a number of volumetric measures, including caudate nucleus size.

In a more recent study, using MRI, Scarone et al. (1992) found increased caudate nucleus volume in the right hemisphere of OCD patients $(n=20)$ relative to controls $(n=16)$, although the OCD patients in this study were all being treated with SSRIs at study. In contrast, Stein et al. (1993), using CT, compared a group of OCD $(n=$ 16) patients and control $(n=8)$ subjects and found no caudate nucleus abnormalities. The authors did note, however, that a subgroup of OCD patients exhibiting high neurological soft signs showed lateral ventricle enlargement.

Using MRI, Calabrese, Colombo, Bonfanti, Scotti, and Scarone (1993) found greater $T_{1}$ signal asymmetry in the 
caudate nuclei of OCD patients relative to controls. This contrasts with Garber, Ananth, Chiu, Griswold, and Oldendorf (1989), who reported equivalent $T_{1}$ signal symmetry in OCD patient and control subject groups. Robinson et al. (1995) found reduced caudate volume in a group of OCD patients relative to controls ( $n=26$ in each group), but did not exclude depressed or medicated OCD patients. Using MRI, Jenike et al. (1996) found a nonsignificant trend toward reduced caudate volume in a group of OCD patients, as well as a significant reduction in total brain white matter, a significant increase in total cortex, and a significant increase in opercular volume. Finally, Aylward et al. (1996) found no significant abnormalities in the lateral ventricles and caudate nuclei of $24 \mathrm{OCD}$ patients relative to controls $(n=21)$.

Although abnormalities have been reported in the lateral ventricles and caudate nucleus, these results are not universal. A number of factors may be responsible for this confusion, including (1) differences in neuroimaging techniques, (2) differences in patient population characteristics, and (3) variable exclusion criteria, particularly with respect to depression and medication. For example, of the three studies in which caudate nucleus abnormalities were reported, in one, the 20 OCD patients had all received medication at the time of scanning (Scarone et al., 1992); in another, medicated and depressed OCD patients were included (Robinson et al., 1995); and in one, the subjects were atypical childhood-onset OCD patients (Luxenberg et al., 1988; see Alyward et al., 1996, for further comment). Attempts to increase methodological consistency across studies will certainly improve the replicability of previous results.

\section{Functional Brain Imaging}

Functional neuroimaging studies of OCD have concentrated on three paradigms: resting-state brain metabolism, symptom provocation, and behavioral or drug treatment interventions. While structural neuroimaging studies have suggested caudal and ventricular abnormalities, functional neuroimaging studies have focused on dysfunctional brain circuits involving the caudate nucleus, the orbital frontal cortex, and the thalamus.

Resting-state brain metabolism. In a number of studies, researchers have looked at baseline differences in metabolic activity between OCD patients and controls. Baxter et al. (1988) used PET to compare 10 nonmedicated OCD patients (with no history of depression) with 10 control subjects matched for age and gender. The authors reported increased overall metabolic activity in the cerebral hemispheres, as well as increased activity in the orbital gyri and caudate nuclei. In two more recent studies, PET revealed increased activity in the orbitofrontal regions, but no differential activity within the caudate nuclei (Nordahl et al., 1989; Swedo, Schapiro, et al., 1989).

Using PET, Martinot et al. (1990) found decreased metabolic activity within the prefrontal lateral cortex, as well as in the striatum and thalamus, in a group of 16 OCD patients. However, 10 of the 16 patients were undergoing drug treatment at the time of the study.

Using SPECT, Machlin et al. (1991) found increased metabolic activity in the medial frontal regions of 10 OCD patients, all of whom were medication free and had no history of major depression. The authors noted that activity in the orbitofrontal regions was not significantly different between patient and control subjects.

In a more recent study, Rubin, Villanueva-Meyer, Ananth, Trajmar, and Mena (1992) employed SPECT to compare groups of OCD and control subjects $(n=10$ in each group) and found increased perfusion in the orbitofrontal cortex bilaterally, as well as decreased metabolic activity bilaterally in the caudate nucleus. Finally, Lucey et al. (1995), using SPECT, compared 30 OCD patients with 30 healthy control subjects and found decreased metabolic activity in the right caudate nucleus and the right thalamus, as well as in several cortical areas, including the right inferior frontal cortex.

Many of the criticisms leveled at structural neuroimaging studies hold true for functional neuroimaging studies of OCD as well. The large number of conflicting reports may be the result of marked differences in patient selection criteria, differences in patient characteristics (e.g., disease onset, symptom subtypes, or gender), or neuroimaging techniques. Nevertheless, abnormal baseline activity in the orbitofrontal cortex, caudate nucleus, and thalamus are suggested in a number of studies.

Symptom provocation. Provocation studies provide a more direct examination of aberrant brain activity associated with obsessive-compulsive episodes. They also allow the OCD patient to serve as his or her own pre-episode control, although in many studies, groups of healthy control subjects have also been employed. Results consistently suggest the involvement of the orbitofrontal cortex and caudate nucleus.

Zohar et al. (1989) scanned a group of OCD subjects, using ${ }^{133} \mathrm{Xe}$ inhalation under one of three conditions: rest, imaginal flooding, or contamination exposure. The results showed that whereas imaginal flooding was associated with increased cerebral blood flow (rCBF) in most cortical regions, actual contaminant exposure was associated with decreased CBF, particularly in the parietal and temporal regions. Using PET, Rauch et al. (1994) found that a symptom-provoked state produced significantly greater brain activation in the right caudate nucleus, in the left anterior cingulate gyrus, and bilaterally in the orbitofrontal cortex. In addition, there was a nonsignificant trend toward greater activation in the thalamus associated with the symptomatic state.

McGuire et al. (1994) also used PET to examine differences associated with symptom-provoked obsessional states. The authors reported increased rCBF in the orbitofrontal cortex and in several regions of the basal ganglia, including the caudate nucleus, the putamum, and the globus pallidus. In addition, increased $\mathrm{rCBF}$ was reported in the thalamus and the cingulate gyrus. Breiter 
et al. (1996) provide the only fMRI study to date of rCBF during symptom provocation. The authors report significant activation in the orbitofrontal, anterior cingulate, and insular cortex, as well as in the right caudate nucleus and left lenticulate.

Symptom provocation studies provide the strongest and most consistent evidence of basal ganglia, orbitofrontal, and thalamic involvement. Cumulatively, the reports demonstrate abnormally elevated activity in the orbitofrontal cortex and caudate nucleus of OCD patients in response to symptomatic episodes. In addition, abnormal activity in the cingulate gyrus and thalamus has been reported in some studies.

Treatment studies. Neuroimaging studies have also been used to examine changes in brain activity associated with behavioral or drug therapy in OCD. These reports, together with symptom provocation studies, provide compelling evidence concerning the dynamic changes in brain activity associated with the ebb and flow of OCD episodes.

The majority of neuroimaging studies of treatment intervention involve drug therapy, with subjects being scanned prior and subsequent to the intervention. Except for the studies done by Hoehn-Saric, Pearlson, Harris, Machlin, and Camargo (1991) and Rubin, Ananth, VillanuevaMeyer, Trajmar, and Mena (1995), who used SPECT, all the studies presented below used PET methodology.

Baxter et al. (1987) treated 10 OCD patients with trazodone (an antidepressant) coupled with an MAO inhibitor and found increased activity in the right caudate nucleus relative to a pretreatment baseline. In a more recent report, Baxter et al. (1992) examined the effects of drug treatment (fluoxetine) and behavioral therapy on rCBF and found that activity in the right caudate nucleus decreased significantly in subjects who responded to treatment, regardless of form (i.e., pharmacological or behavioral). In addition, activity was significantly correlated in the orbital frontal cortex, caudate nucleus, and thalamus before, but not after, successful treatment. Schwartz, Stoessel, Baxter, Martin, and Phelps (1996) also examined the effects of behavioral modification on brain metabolism and found decreased metabolic rates in the caudate nucleus bilaterally in OCD responders. The authors also found that correlated activity in the caudate nucleus, orbital gyri, and thalamus decreased significantly after treatment.

Other groups have looked at the effects of SSRIs on brain activity in OCD patients. Benkelfat, Nordahl, Semple, and King (1990) found decreased activity in the left caudate nucleus and right orbitofrontal cortex following a 16-week trial of clomipramine. Hoen-Saric, Pearlson, et al. (1991) found decreased activity in the medial-frontal cortex following a 12- to 16-week trial of fluoxetine. Swedo et al. (1992) found decreased activity in the orbitofrontal cortices (both left and right), but not caudate nucleus, after 1 year of clomipramine treatment. Perani et al. (1995) treated 11 patients with fluvoxamine, fluoxetine, or clomipramine and found decreased activity in the cingulate cortex after 12 weeks. Rubin et al. (1995) also examined the effects of clomipramine on $\mathrm{rCBF}$ and found decreased metabolic activity in the orbitofrontal, posterofrontal, and dorsal parietal regions following treatment.

Treatment studies suggest that changes in the $\mathrm{OC}$ experience that result from pharmacological or behavioral intervention are accompanied by corresponding decreases in regional $\mathrm{CBF}$ in certain brain regions, including the orbitofrontal cortex, caudate nucleus, and thalamus. These results complement symptom-provocation studies, which have consistently shown increased activity in these same regions in response to obsessive or compulsive episodes. Despite differences in neuroimaging techniques, patient characteristics (e.g., gender, disease onset, and symptom subtypes), and exclusion criteria, neuroimaging studies of OCD indicate a number of brain regions that seem to be intimately involved in OCD expression, including the orbitofrontal cortex, the basal ganglia and the thalamus.

\section{NEUROPSYCHOLOGY OF OCD}

In the last 15 years, efforts to characterize the cognitive neuropsychology of OCD have been quite successful. Although neuropsychological testing provides a less direct examination of the neuropathology of OCD than does neuroimaging, the results provide complementary information. Neuropsychological testing also allows researchers to document the range and severity of cognitive dysfunction in OCD patients. Indeed, a broader understanding of the cognitive manifestations of the disorder (i.e., beyond the cognitive deficits most evident during symptom expression) will be critical for establishing a cognitive neuroscience of OCD.

Neuropsychological testing is currently aimed at multiple levels of cognitive function, ranging from more "basic" sensorimotor programming to the highest level "executive" functions. Whereas many of the preliminary investigations were inspired by the paucity of results in this area, more recent efforts have been motivated by specific predictions based on neuroanatomical considerations. This has resulted in a complement of studies that not only catalogue the neuropsychological profile of OCD, but that provide critical empirical tests for current models of OCD.

\section{Sensorimotor and Attentional Mechanisms}

Cognitive deficits in OCD occur quite early in both the sensory and motor pathways. Deficits in sensory input gating (Swerdlow, Benbow, Zisook, Geyer, \& Braff, 1993) as well as in saccadic eye movement programming (Sweeney, Palumbo, Halper, \& Shear, 1992; Tien, Pearlson, Machlin, Bylsma, \& Hoehn-Saric, 1992) have both been established in OCD. Several reports have also demonstrated abnormal attentional mechanisms in OCD patients (e.g., Towey et al., 1994). Although it is true that saccadic programming, sensorimotor gating, and attention have all been linked to orbitofrontal cortex and basal ganglia function (and thus provide confirmation of neuro- 
imaging results), interpretation of these results with respect to OCD symptomology is not at all clear. Oculomotor programming deficits provide an excellent example.

Proper oculomotor (e.g., saccadic) programming relies on normal basal ganglia function. Diseases of the basal ganglia, such as Huntington's disease, have been shown to disrupt oculomotor programming (Cummings, 1993). Given the ability of Huntington's disease to induce OCD, and given the establishment (through neuroimaging) of basal ganglia dysfunction in OCD (e.g., Baxter, Schwartz, Guze, Bergman, \& Szuba, 1990), similar oculomotor deficits might be expected in OCD patients. Tien et al. (1992) and Sweeney et al. (1992) each describe a series of experiments designed to test this hypothesis. Tien et al. reported that OCD subjects showed greater error rates and greater numbers of inaccurate saccades in a goal-directed antisaccade task. There was no difference, however, in overall reaction time, saccadic velocity, or accuracy of saccades in a visually guided saccade task. The authors conclude that this performance is consistent with disruption of basal ganglia function, but they offer no connection between oculomotor programming deficits and OCD symptomology.

Sweeney et al. (1992) reported similar results, with OCD patients having difficulty in smooth tracking of targets, an increased rate of "reversal saccades" to compensate for target overshooting, and an increased rate of "square-wave" (intrusive) saccades while attempting to maintain fixation. The authors concluded that the results were consistent with dysfunction in the basal ganglia and frontal regions, but no connection was made between these results and OCD symptom expression.

The preceding examples are typical of neuropsychological testing in OCD: Strides in documenting neuroanatomical, cognitive, and behavioral deficits have not necessarily shed light on the mechanisms that produce the disorder. To be sure, not all deficits witnessed in OCD are fundamentally involved in the expression of the disorder. Indeed, some deficits co-occur because of shared neural substrates. This is perhaps the greatest challenge in constructing an adequate account of the mechanisms of OCD: knowing which deficits are intimately related to OCD expression and which are merely concomitant.

More relevant to OCD symptomology, Swerdlow et al. (1993) hypothesized that the inability of OCD patients to gate inappropriate thoughts (obsessions) may reflect a more central sensorimotor gating deficit. Their dependent measure was the prepulse inhibition (PPI) of a startle reflex. Normally, a startle response can be suppressed when the startling stimulus is preceded by a weak "prepulse." Deficient sensorimotor gating, however, reduces the PPI. Striatal dysfunction could produce such a deficit, since this area is known to be important in the normal suppression of the startle reflex, and interestingly, the striatal regions have been implicated in OCD pathology. Thus, the underlying neuroanatomy of OCD may produce a general deficit in gating sensorimotor stimuli.

Swerdlow et al. (1993) reported that OCD patients did in fact show a deficit in sensorimotor gating, as mea- sured by a reduction in PPI, but they were careful to note that the relationship between their results and the inability of OCD patients to inhibit obsessional thoughts is unclear. That is, whether a general deficit in sensorimotor gating produces, or simply accompanies, the inability to suppress the particular obsessions experienced by OCD patients remains uncertain. Their findings nevertheless appear relevant to the cognitive and behavioral phenomenology of OCD.

Swerdlow et al.'s (1993) findings reflect, perhaps, an early attentional disturbance in OCD patients. Nelson, Early, and Haller (1993) conducted a more direct examination of the attentional processes of $O C D$ patients using a Posnerian attention task (Posner, Nissen, \& Ogden, 1978). In this task, a central fixation cross is flanked by two target locations. Subjects are required to respond to a target in either location as quickly as possible. On each trial, target presentation is preceded by a location cue that is valid, invalid, or noninformative (neutral). Typically, responses are faster in the valid cue condition than in the neutral condition. Similarly, responses are faster in the neutral condition than in the invalid condition.

Although the performance of OCD patients was in most respect equivalent to that of control subjects, there was a marked difference in their inhibition of return. Inhibition of return refers to the finding that valid precuing normally produces benefits in reaction time, long intervals between valid cue and target presentation actually produce a cost. This inhibition of return is a common finding in the Posnerian attentional task, and it reflects, perhaps, an inherent preference for novelty. In Nelson et al.'s (1993) study, unlike control subjects who exhibited normal inhibition of return, OCD patients showed a decreased inhibition of return for targets presented in the left visual field, and no inhibition of return for targets presented in the right visual field.

Nelson et al. (1993) interpret their results as a disruption of the "novelty bias" in OCD patients, which could reflect a general impairment in the ability to suppress attended stimuli, such as obsessional thoughts. They conclude that this deficit may reflect a "breakdown of the gating mechanism allowing normally ignored, exogenous stimuli to occupy endogenous resources" (p. 194). Clearly, this finding suggests a possible mechanism by which obsessional thoughts fail to be suppressed in OCD patients, but again, any such mechanism is strictly speculative. Furthermore, this account does not address the specificity of obsessional thoughts--a qualification which will resurface repeatedly in this examination. That is, a general impairment in the ability to suppress "normally ignored, exogenous stimuli" does not explain the marked specificity of obsessional thoughts, both within (i.e., patients normally experience only one thought obsessionally) and across (i.e., the range of obsessional thoughts is remarkably narrow) patients.

The examination of sensorimotor and basic attentional mechanisms in OCD patients has been helpful in confirming the involvement of certain brain regions but has contributed relatively little to our knowledge of the na- 
ture of OCD symptoms. Analysis of higher cognitive functions may prove more helpful in establishing such a link. Nevertheless, any indication of higher level cognitive dysfunction must be interpreted with respect to hyperactivation of attentional mechanisms.

\section{Traditional Neuropsychological Testing}

Two results appear consistently across studies of higher level cognitive functions in OCD patients - namely, impairments in visuospatial and cognitive set-shifting abilities. The relationship between these findings and possible deficits in more basic attentional mechanisms remains unclear. It should be noted that for many of the measures discussed below, both positive and negative results have been reported (e.g., OCD patient performance on the Wisconsin Card Sorting Task [WCST] has been reported as impaired [Head, Bolton, \& Hymas, 1989] and as within normal ranges [Abbruzzese, Bellodi, Ferri, \& Scarone, 1995], depending on the study). Similar disagreement about laterality of impairment also exists (e.g., primarily left hemisphere [Flor-Henry, Yeudall, Koles, \& Howarth, 1979] involvement in some reports, and primarily right hemisphere [Insel et al., 1983] involvement in others). Nevertheless, several common themes have emerged.

Flor-Henry et al. (1979) offered perhaps the first extensive examination of neuropsychological test performance in OCD. The research was motivated largely by the lack of clear documentation of cognitive deficits in OCD patients. The authors compared the performance of OCD patients with age- and education-matched controls, on tests of spatial abilities, frontal lobe functioning, verbal fluency, and a range of similar tasks. Although they found no significant difference on tasks such as Verbal Fluency and the Vocabulary and Block Design subtests of the WAIS, they did note differences on others tasks such as Raven's Coloured Progressive Matrices, tactual performance tasks, the Purdue Pegboard task, and the Digit Span and Digit Symbol subtests of the WAIS. The authors argued that this pattern of difficulties most likely reflected frontal dysfunction, primarily in the left hemisphere.

Several of Flor-Henry et al.'s (1979) initial findings were not consistently replicated. Insel et al. (1983), for instance, attempted to replicate the results with a group of 18 OCD patients. Results from only 1 of 11 neuropsychological tests (a nonvisual spatial organization test) were replicated, although it must be noted that Insel et al. used normative data, not control subjects, in their replication study. The authors argued that their findings were more accurately represented as a deficit in spatial perception, and therefore more consistent with right, rather than left, hemisphere damage.

Behar et al. (1984) conducted a similar study and found that OCD patients were impaired on several visuospatial tasks, including the Stylus Maze task, the Money Road Map Test (MRMT), and the Rey-Osterrieth complex figure task. The authors suggested a visuospatial deficit and right-hemisphere asymmetry but also highlighted the role of the frontal cortex.

In an attempt to address the discrepancies between previous findings, Head et al. (1989) administered several tests aimed at assessing the visuospatial and set-shifting abilities of 15 OCD patients. The authors used the Modified Wisconsin Card Sorting Task (MWCST) to assess set-shifting ability, the Block Design subtest of the WAIS (WAIS-BD) to assess spatial abilities, and the MRMT to assess both set-shifting and visuospatial abilities within the same task. Reliable differences on the MWCST, the WAIS-BD, and the MRMT were found, leading the authors to propose that OCD patients had difficulties in nonverbal tasks that required set-shifting. Results of more general tests of set-shifting and visuospatial skills were equivocal, leading the authors to withhold any conclusions about more general impairments in either domain.

The results of early efforts to capture the neuropsychological profile of OCD patients were inconsistent in many cases. Researchers in more recent studies have attempted to address these inconsistencies and in some cases have provided plausible explanations for previous discrepancies. For example, Martinot et al. (1990) noted inconsistencies with respect to laterality of impairment and frontal lobe functioning in OCD patients. They argued that these discrepancies could be due to poor experimental control of several factors: (1) the use of depressed versus nondepressed OCD patients, (2) medicated versus medication-free patients, and ( 3 ) control subjects versus normative data. They therefore compared the performance of nondepressed OCD patients (using medicated and medication-free subgroups) with age-matched control subjects on a variety of neuropsychological tests.

Five general areas of cognitive function-namely, language, mental control, global memory, attention, and praxis - were tested (Martinot et al., 1990). Language abilities, assessed by tests of verbal fluency, appeared normal as did performance on several tests of attentional abilities, including the Trail Making and Graphic Alternating Sequence tasks. The authors did, however, find impairment in the Stroop task as well as several memory tasks, including the Rey Figure and Rey Verbal Learning tasks. The authors concluded that with experimental control of the considerations mentioned above, OCD patients showed a selective attentional deficit.

Boone, Ananth, Philpott, Kaur, and Djenderedjian (1991) conducted a similar study addressing the following cognitive areas: visual spatial skills, memory and attention, frontal lobe functioning, and intelligence. They found no significant differences between 20 nondepressed OCD patients and control subjects on tests of intelligence, attention, verbal memory, or frontal lobe tasks. Interestingly, the frontal lobe tasks included the WCST. They did, however, note significant deficits on several nonverbal visual memory tasks, such as the Hooper Visual Organization and Rey-Osterrieth Complex Figure tasks. The authors concluded that these impairments were consis- 
tent with basal ganglia dysfunction, and possibly right hemisphere laterality.

The apparent disagreement concerning frontal lobe functioning led Hoehn-Saric, Harris, et al. (1991) to examine the possibly causal role of pharmacological treatment in the development of frontal syndrome symptoms in OCD. They, like Martinot et al. (1990), were concerned with poor experimental control in previous reports, specifically in the use of medicated patients. The authors hypothesized that fluoxetine, a commonly prescribed drug in the treatment of $O C D$, produced symptoms that were characteristic of frontal lobe syndrome. Thus, deficient performance by OCD patients in frontally mediated neuropsychological tests may have resulted from the treatment of these patients, rather than from the disorder itself.

Hoen-Saric, Harris, et al. (1991) administered a series of neuropsychological tests to an OCD patient before and after treatment with fluoxetine. The subject scored in the normal range on all tests prior to medication, including Verbal and Design Fluency, Complex Figure Copy and Recall, Verbal Learning, Stroop, and Trail Making tasks. Performance on tests of sustained attention and inhibition of prepotent responses (the Stroop and Trail Making tasks) were unaffected during treatment. Performance on tasks that involved the generation of novel responses, set-shifting, and memory were all impaired during the administration of fluoxetine. The authors argued that symptoms of frontal lobe syndrome were not intrinsic to the disorder, but rather were the result of pharmacological intervention. The authors argue in conclusion that this result is capable of explaining several inconsistencies in previous research.

Another group (Christensen, Kim, Dysken, \& Hoover, 1992) examined the neuropsychological functions of 18 OCD patients specifically in the areas of verbal abilities, visual spatial abilities, recent verbal memory, recent nonverbal memory, and executive function. The authors found deficits in tests of recent nonverbal memory, as well as visuospatial reasoning. They did not, however, find deficits in tasks involving executive functions, verbal abilities, or recent verbal memory. Although they are careful to note that speeded performance had a profound impact on a variety of tasks, the most obvious deficiency was within the domain of recent nonverbal memory. They argue further that this pattern of impairment is consistent with damage to mesial temporal areas of the right hemisphere, possibly in the limbic and paralimbic regions.

In many of the studies presented above, the performance of OCD patients was assessed on a wide variety of neuropsychological tasks without a specific model of etiology. In more recent examinations, driven by specific theories of OCD etiology, researchers have attempted to ask more focused questions about performance on specific tasks. These attempts have also focused more directly on the relationship between symptom expression and neuropsychological test performance, with the former often generating predictions for the latter. Two examples are presented below.
Brown, Kosslyn, Breiter, Baer, and Jenike (1994) examined the performance of OCD patients on several tasks under the assumptions of signal detection theory. They tested a group of OCD patients on a task that required discrimination of real and imagined words, with the hypothesis that OCD patients are less capable of discriminating between real and imagined events. That is, they hypothesized that OCD patients are more greatly impaired in discriminating between actually performing an action as opposed to only imaging it, and that this inability underlies OCD symptom expression. Contrary to their predictions, however, OCD patients showed greater ability to discriminate between real and imaged events in a signal detection task, as measured both by subjects' sensitivity to stimuli $\left(d^{\prime}\right)$ and by criteria for decision $(\beta)$.

Abbruzzese et al. (1995) conducted a more recent investigation of cognitive neuropsychological test performance in OCD patients. The authors hypothesized that if orbitofrontal cortex (OFC), as opposed to dorsolateral prefrontal cortex (DLPFC) was implicated in OCD, patients should show deficits on OFC-dependent tasks (such as the object alternation tasks, or OAT), but not on DLPFC-dependent tasks (such as the WCST). Consistent with their hypothesis, Abbruzzese et al. found that 25 OCD patients were impaired on the OAT but not the WCST. As a control, they also found that 25 schizophrenic patients, who are presumed to have only DLPFC damage, were impaired on the WCST but not the OAT.

Taken together, neuropsychological testing has suggested several problems in OCD: hyperactivation of attentional systems, difficulties with cognitive set-shifting, and visuospatial deficits. In contrast, general intelligence, verbal skills, and executive functions (with the exception of set-shifting) all remain preserved in OCD.

\section{MODELS OF OCD}

Models of OCD expression have focused on a number of issues, including the production and execution of goaldirected motor programs, malfunction of behavioral inhibition systems, and the release of species-specific adaptive behaviors. Although many of these models account for the general characteristics of OCD, they often lack the requisite specificity to explain the very constrained nature of OCD symptomology.

Pitman (1987) draws on cybernetic research to develop the notion of a malfunctioning homeostatic system in OCD, using the analogy of a thermostat. Accordingly, perceptual systems are compared with internal reference signals, and an error signal denotes deviation of the former from the latter. The system, experiencing this error signal, recruits the behavioral output system in an attempt to reduce the discrepancy between the actual and desired states.

OCD is thought to result from persistent, high error signals in this model (Pitman, 1987). These error signals arise from several possible malfunctions: Internal conflict between two control systems, intrinsic comparator 
malfunction, and attentional disturbances could each produce unwarranted error signals. According to the theory, these signals are manifested as obsessional thoughts which drive the behavioral output system into effecting compulsive behaviors. Despite the compulsive behaviors, however, the high error signal is never sufficiently reduced.

Pitman (1987) notes that the basal ganglia have been implicated in the production of behavioral (motor) output, and that the limbic system can be viewed as an "internal comparator" within the goal-directed behavioral system, thereby providing a neuroanatomical basis to his theory. He does not, however, provide an explicit role for the orbitofrontal cortex, nor does he address the specificity with which obsessive thoughts appear.

Another model of OCD symptom expression is presented by Baxter et al. (1990), who argue for the role of the striatum in the production of obsessive symptoms. The claim is that "striatal dysfunction may lead to adventitious thoughts and sensations and actions that are normally suppressed with little conscious effort by sitespecific regions of the striatum" (p. 24). Hyperactivity within the orbitofrontal regions, it is argued, reflects the recruitment of cortical mechanisms in an (unsuccessful) attempt to inhibit these thoughts. The authors highlight the similarity between OCD patients, who are unable to prevent intrusion of obsessional thoughts, and Huntington's patients, who are unable to prevent intrusion of unwanted motor programs. This similarity may reflect an overlap in basal ganglia dysfunction in both groups.

Although concerned with the same neuroanatomical circuits as Baxter et al. (1990), Rapoport (1991) offers a very different model of OCD symptomology. On the basis of animal research addressing the effects of caudate nucleus lesions, Rapoport argues that compulsive behaviors are experienced before obsessional thoughts in OCD, and that the latter are undertaken as justifications for the inappropriate nature of the former. The theory assumes that damage to the caudate nucleus releases an excess of certain species-specific, fixed-action patterns that have evolutionary significance. An example is grooming behavior, which in many lower species (as well as our own) is crucial to survival. Such "species-typical self-protective behaviors" (p. 7) are normally regulated by the circuits connecting regions of the cortex, thalamus, and basal ganglia. Dysfunction within this system (particularly in the caudate nucleus) causes excessive release of such behaviors, which are characterized as compulsive behaviors. According to the theory, obsessional thoughts arise as a justification for the inappropriate behaviors in which the OCD patient engages.

Rapoport (1991) offers several examples of analogous behavioral phenomena in lower animals. For example, acral lick dermatitis (in dogs) and excessive preening (in birds) are examples of excessive and ritualistic behaviors performed in a manner similar to the rituals of OCD patients. Interestingly, Rapoport demonstrates the effectiveness of pharmacological agents, specifically SSRIs, in the treatment of such behavioral problems in lower animals, suggesting some consistency across species.

While Rapoport (1991) more directly addresses the specificity and family resemblance of obsessional thoughts and compulsive behaviors, the theory lacks evidence for the described temporal relationship in humans. Furthermore, it cannot account for OCD cases in which the compulsive behaviors are not externally observable, evolutionarily adaptive behaviors, such as prayer, counting, and checking in some cases (e.g., a patient who obsesses about hitting a pedestrian while driving, and who retraces his route while driving to check for such occurrences).

Researchers are beginning to consider the importance of information processing and cognitive dysfunction in the origin of OCD. Stein and Hollander $(1992,1994)$, for example, have argued for the importance of adopting a cognitive science approach to OCD, combining results from a variety of disciplines in explaining how the cognitive as well as the behavioral symptoms of OCD arise. They argue that connectionist simulations of OCD may provide crucial insights into the role of information processing in the emergence of OCD. They also note the success of connectionist modeling in other areas of psychopathology research (e.g., Cohen \& Servan-Schreiber's [1992] connectionist model relating the biological and cognitive deficits in schizophrenia) and appeal for similar models in OCD research.

\section{DISCUSSION}

Researchers have made tremendous progress in understanding OCD over the past decade, particularly with respect to the neuroanatomy and neurophysiology of the disorder. Nevertheless, a major stumbling block is the inability to consistently replicate findings, in both the neuroimaging and the neuropsychological testing literature.

The failure to consistently replicate findings in OCD research may result from a number of methodological differences between studies, including differences in the criteria for excluding medicated or depressed OCD patients. Differential results may also reflect differences in the underlying neuropathology of OCD subgroups. Specifically, it is possible that distinct $O C D$ patient subgroups manifest different impairments within the circuit connecting the orbitofrontal cortex, the basal ganglia, and thalamus. Although aberrant brain activity during OCD episodes likely occurs throughout this circuit, the sources of the dysfunction might be different for different patients. These differences would show up most clearly in structural neuroimaging or baseline functional neuroimaging studies, when the circuit is not actively engaged.

In recent theories, researchers have attempted to divide OCD patients into obsessive and compulsive subgroups (Baer, 1994; Hantouche \& Lancrenon, 1996). On the basis of symptomology and OCD inventory scores, these studies have tended to place patients into one of three groups: purely obsessional (i.e., patients with excessive sexual, religious, or violent imagery), purely com- 
pulsive (i.e., patients with counting or checking rituals as well as patients with symmetry or order obsessions), and mixed (i.e., patients with contamination fears or excessive washing or grooming). It is possible that these subgroups exhibit different neuropathologies within the orbitofrontal-basal-thalamic-cortical circuit, and that these differences underlie some of the inconsistencies within the OCD literature.

Lesion studies have demonstrated that damage to the orbitofrontal cortex produces disinhibition, inappropriate behavior, or emotional displays; inappropriate responses to environmental cues; and utilization behavior (Cummings, Gosenfeld, Houlihan, \& McCaffrey, 1983; Kolb, 1977; Lhermitte, Pillon, \& Serdaru, 1986; Logue, Durward, Pratt, Piercy, \& Nixon, 1968; Rolls, 1985). OCD patients with purely obsessional or purely compulsive symptoms display such characteristics. The inability to inhibit thought patterns, particularly deviant or inappropriate ones, is characteristic of many of these patients. In addition, checking, counting, and hoarding rituals can all be seen as a type of utilization behavior or as an inappropriate response to environmental cues. These two OCD patient subgroups therefore share much in common with "orbitofrontal" patients.

Neurological studies have demonstrated the link between damage to the basal ganglia and compulsive behaviors in a number of excessive motor disorders, including Sydenham's chorea (Swedo, Rapoport, et al., 1989), Huntington's disease (Cummings \& Cunningham, 1992), and Tourette's syndrome (Insel, 1992). Rapoport has similarly reported that the basal ganglia may be involved in disorders of species-specific grooming or washing behaviors such as excessive hair pulling (trichotillomania) and excessive nail biting in humans, and acral dermatitis or excessive preening in animals (Rapoport, 1991). OCD patients with fears of contamination or washing and grooming compulsions (i.e., the "mixed" variety) can similarly be characterized as having dysfunctional grooming or washing behaviors and therefore share much in common with these "basal ganglia" patients.

Although OCD episodes most likely result from aberrant activity within the entire frontal-basal-thalamic loop, the origin of abnormal activity may be different in different cases. OCD symptoms may be triggered by dysfunctional orbitofrontal activity in some cases and by dysfunctional basal ganglia activity in others. Thus, brain imaging or neuropsychological testing may find different patterns, depending on the source of dysfunction. If the patient group draws more heavily from one group than from another, we might expect to find differences in the observed neuropathology. That is, resting-state metabolic studies and structural neuroimaging studies may find a greater incidence of orbital frontal deficits in OCD patients who experience purely obsessional or purely compulsive symptoms and a greater incidence of basal ganglia dysfunction in OCD patients with mixed symptomology (i.e., those with contamination fears or washing and grooming rituals). Unfortunately, relatively few neuro- imaging studies report the specific symptoms experienced by subjects (with the notable exception of symptom provocation studies).

Potential differences in the underlying neuropathology among OCD subtypes is one of a number of potential confounds in current OCD research. The effects of medication, the inclusion of depressed OCD patients, gender differences, and differences between childhood- and adultonset OCD also must be considered in interpreting the results of previous investigations. Until these factors are systematically explored in an empirical manner, any theory of OCD neuropathology and symptom expression will be premature.

Our review of the neuroanatomy and neuropsychology of OCD suggests several directions for further research. One priority is a more systematic exploration of several patient selection criteria, including the use of medication, the exclusion of depressed OCD patients, the age of disease onset, the patient's gender, and the symptoms experienced by each subject. Greater uniformity in selection criteria across study will no doubt improve the replicability of previous results. Specifically, attention to these details will confirm the precise role of the orbitofrontal cortex, the basal ganglia, and the thalamus in OCD expression.

Another important issue in OCD research is that of understanding the role of set-shifting impairments and hyperattention in the emergence of OCD. That is, are the cognitive impairments causally related to $\mathrm{OCD}$, or vice versa, or do both emerge simultaneously? It seems likely that the two reflect a common underlying mechanism, but this mechanism has not been studied experimentally. Furthermore, serious attempts to relate visuospatial deficits to OCD phenomenology are needed. Visuospatial deficits have been considered only in light of what they may tell us about possible underlying neuroanatomy. A more complete analysis, however, with examination of the relationship between this deficit and OCD symptomology seems imperative.

One of the most difficult features of OCD, and one for which little has been said, is the rather restricted nature of obsessional thoughts. Although it is possible to conceive of obsessions taking many forms, remarkably few types have been reported. An adequate theory must account for this restricted class of potential obsessions and characterize the family resemblance they share. Few theories have attempted to describe these characteristics, but cognitive neuroscience may uncover valuable clues about the nature and basis of obsessional thoughts in particular, and OCD in general.

\section{CONCLUSIONS}

The question still remains: By what brain mechanisms do the particular symptoms experienced by the OCD patient arise? Although research on OCD has flourished at many levels, a successful integration of this information is still lacking. Before any such integration can be fruit- 
ful, however, several aspects of OCD research methodology need to be addressed systematically. In this review, it is suggested that medication, depression, gender, disease onset, and symptom subtype may all conspire to produce differential results, particularly in the neuroimaging literature. Systematic exploration of these factors will reveal the true neurobiological factors relevant to OCD expression, and only then will an adequate theory of OCD emerge.

Greater concentration on the cognitive aspects of $O C D$, and specifically on the information-processing characteristics, is also necessary. Although many of the currently popular models take aim at the production of compulsive behaviors, a more focused examination of the nature and inception of obsessional thoughts is equally important. It seems imperative, therefore, that greater emphasis be placed on understanding the nature of obsessional thoughts and on their emergence within the framework of general cognition.

Substantial evidence has been gathered concerning the biological and cognitive foundations of OCD, but our task is far from complete. Functional neuroimaging has implicated the orbitofrontal cortex, basal ganglia, and thalamus in the expression of OCD. Neuropsychological studies have highlighted impairments in cognitive setshifting, hyperactivation of attentional systems, and visuospatial deficits. These findings offer guidance for constructing a cognitive neuroscience of OCD, but further work will be necessary before any such theory is possible.

Our knowledge of the nature of OCD is growing rapidly. Neurobiological studies of OCD, although relatively recent, have made great strides. To be sure, only through further such empirical investigation will an adequate account of $O C D$ emerge. It can be concluded, however, that a greater emphasis on methodological consistency and an integration of results from neuroimaging and neuropsychological testing will be critical before any such account can be possible.

\section{REFERENCES}

Abbruzzese, M., Bellodi, L., Ferri, S., \& Scarone, S. (1995). Memory functioning in obsessive-compulsive disorder. Behavioral Neurology, 6, 119-122.

American Psychological Association (1994). Diagnostic and statistical manual of mental disorders (4th ed.). Washington, DC: Author

aylward, E. H., Harris, G. J., Hoehn-Saric, R., Barta, P. E., MachLIN, S. R., \& Pearlson, G. D. (1996). Normal caudate nucleus in obsessive-compulsive disorder assessed by quantitative neuroimaging. Archives of General Psychiatry, 53, 577-584.

BAER, L. (1994). Factor analysis of symptom subtypes of obsessive compulsive disorder and their relation to personality and tic disorders. Journal of Clinical Psychiatry, 55, 18-23.

BARLOW, D. H. (1988). Anxiety and its disorders: The nature and treatment on anxiety and panic. New York: Guilford Press.

Baxter, L. R., Schwartz, J. M., Bergman, K. S., Szuba, M. P., Guze, B. H., Mazziotta, J. C., Alazraki, A., Selin, C. E., Ferng, H.-K., Munford, P., \& Phelps, M. E. (1992). Caudate glucose metabolic rate changes with both drug and behavior therapy for obsessive- compulsive disorder. Archives of General Psychiatry, 49, 681-689.

Baxter, L. R., Schwartz, J. M., Guze, B. H., Bergman, K. S., \& SzuBA, M. P. (1990). Neuroimaging in obsessive-compulsive disorder: Seeking the mediating neuroanatomy. In M. Jenike, L. Baer, \& W. Minichiello (Eds.), Obsessive-compulsive disorders: Theory \& management (pp. 167-188). St. Louis: Mosby.

Baxter, L. R., Schwartz, J. M., Mazziotta, J. C., Phelps, M. E. Pahl, J. J., Guze, B. H., \& Fairbanks, L. (1988). Cerebral glucose metabolic rates in nondepressed patients with obsessive-compulsive disorder. American Journal of Psychiatry, 145, 1560-1563.

Baxter, L. R., Thompson, J. M., Schwartz, J. M., Guze, B. H., Phelps, M. E., Mazziotta, J. C., Selin, C. E., \& Moss, L. (1987). Trazodone treatment response in obsessive-compulsive disordercorrelated with shifts in glucose metabolism in the caudate nuclei. Psychopathology, 20(Suppl. 1), 114-122.

Behar, D., Rapoport, J. L., Berg, C. J., Denckla, M. B., ManN, L., Cox, C., Fedio, P., ZahN, T., \& Wolfman, M. G. (1984). Computerized tomography and neuropsychological test measures in adolescents with obsessive-compulsive disorder. American Journal of Psychiatry, 141, 363-369.

Benkelfat, C., Nordahl, T. E., Semple, W. E., \& King, A. (1990). Local cerebral glucose metabolic rates in obsessive-compulsive disorder: Patients treated with clomipramine. Archives of General Psychiatry, 47, 840-848.

BLACK, A. (1974). The natural history of obsessional neurosis. In H. R. Beech (Ed.), Obsessional states (pp. 19-54). London: Methuen.

Boone, K. B., Ananth, J., Philpott, L., Kaur, A., \& DjenderedJIAN, A. (1991). Neuropsychological characteristics of nondepressed adults with obsessive-compulsive disorder. Neuropsychiatry, Neuropsychology, \& Behavioral Neurology, 4, 96-109.

Breiter, H. C., Rauch, S. L., Kwong, K. K., Baker, J. R., Weisskoff, R. M., Kennedy, D. N., Kendrick, A. D., Davis, T. L., Jiang, A., Cohen, M. S., Stern, C. E., Belliveau, J. W., Baer, L., O'Sullivan, R. L., Savage, C. R., Jenike, M. A., \& Rosen, B. R. (1996). Functional magnetic resonance imaging of symptom provocation in obsessive-compulsive disorder. Archives of General Psychiatry, 53, 595-606.

Brown, H. D., Kosslyn, S. M., Breiter, H. C., BaEr, L., \& Jenike, M. A. (1994). Can patients with obsessive-compulsive disorder discriminate between percepts and mental images? A signal detection analysis. Journal of Abnormal Psychology, 103, 445-454.

Calabrese, G., Colombo, C., Bonfanti, A., Scotti, G., \& Scarone, S. (1993). Caudate nucleus abnormalities in obsessive-compulsive disorder: Measurements of MRI signal intensity. Psychiatry Research: Neuroimaging, 50, 89-92.

Christensen, K. J., Kim, S. W., Dysken, M. W., \& Hoover, K. M. (1992). Neuropsychological performance in obsessive-compulsive disorder. Biological Psychiatry, 31, 4-18.

Cohen, J. D., \& Servan-Schreiber, D. (1992). Context, cortex, and dopamine: A connectionist approach to behavior and biology in schizophrenia. Psychological Review, 99, 45-77.

Cummings, J. L. (1993). Frontal-subcortical circuits and human behavior. Archives of Neurology, 50, 873-880.

Cummings, J. L., \& Cunningham, K. (1992). Obsessive-compulsive disorder in Huntington's disease. Biological Psychiatry, 31, 263-270.

Cummings, J. L., Gosenfeld, L. F., Houlihan, J. P., \& McCaffrey, T. (1983). Neuropsychiatric disturbances associated with idiopathic calcification of the basal ganglia. Biological Psychiatry, 18, 591-601.

FENICHEL, O. (1945). The psychoanalytic theory of neurosis. New York: Norton.

Flor-Henry, P., Yeudall, L. T., Koles, Z. J., \& Howarth, B. G. (1979). Neuropsychological and power spectral EEG investigations of the obsessive-compulsive syndrome. Biological Psychiatry, 14, 119.130 .

Garber, H. J., Ananth, J. V., Chiu, L. C., Griswold, V. J., \& OldenDORF, W. H. (1989). Nuclear magnetic resonance study of obsessivecompulsive disorder. American Journal of Psychiatry, 146, 1001-1005.

Hantouche, E. G., \& LANCrenon, S. (1996). Typologie moderne des symptomes et des syndromes obsessionnels-compulsifs: Résultats 
[Modern typology of obsessive-compulsive symptoms and syndromes: Results]. Encephale, 22, 9-21.

Head, D., Bolton, D., \& Hymas, N. (1989). Deficit in cognitive shifting ability in patients with obsessive-compulsive disorder. Biological Psychiatry, 25, 929-937.

Hembree, E. A., FoA, E. B., \& KozaK, M. J. (1994). Obsessivecompulsive disorder. In B. B. Wolman \& G. Stricker (Eds.), Anxiety and related disorders: $A$ handbook (pp. 177-193). New York: Wiley.

Hoehn-Saric, R., Harris, G. J., Pearlson, G. D., Cox, C. S., MachLIN, S. R., \& CAMARGo, E. E. (1991). A fluoxetine-induced frontal lobe syndrome in an obsessive compulsive patient. Journal of Clinical Psychiatry, 52, 131-133.

Hoenn-Saric, R., Pearlson, G. D., Harris, G. J., Machlin, S. R., \& Camargo, E. E. (1991). Effects of fluoxetine on regional cerebral blood flow in obsessive-compulsive patients. American Journal of Psychiatry, 148, 1243-1245.

INSEL, T. R. (1992). Toward a neuroanatomy of obsessive-compulsive disorder. Archives of General Psychiatry, 49, 739-744.

Insel, T. R., Donnelly, E. F., Lalakea, M. L., Alterman, I. S., \& MURPHY, D. L. (1983). Neurological and neuropsychological studies of patients with obsessive-compulsive disorder. Biological Psychiatry, 18, 741-751.

Jenike, M. A., Baer, L., Ballantine, T., Martuza, R. L., Tynes, S., GiRIUNAS, I., BUTTOLPH, M. L., \& CASSEM, N. H. (1991). Cingulotomy for refractory obsessive-compulsive disorder: A long-term follow-up of 33 patients. Archives of General Psychiatry, 48, 548-555.

Jenike, M. A., Breiter, H. C., Baer, L., Kennedy, D. N., Savage, C. R., Olivares, M. J., O'Sullivan, R. L., Shera, D. M., Rauch, S. L., Keuthen, N., Rosen, B. R., Caviness, V. S., \& Filipek, P. A (1996). Cerebral structural abnormalities in obsessive-compulsive disorder. Archives of General Psychiatry, 53, 625-632.

Kellner, C. H., Jolley, R. R., Holgate, R. C., Austin, L., Lydiard, R. B., LARAiA, M., \& BALlenger, J. C. (1991). Brain MRI in obsessivecompulsive disorder. Psychiatry Research, 36, 45-49.

KoLB, B. (1977). Studies on the caudate-putamen and then dorsomedial thalamic nucleus of the rat: Implications for mammalian frontal-lobe functions. Physiology \& Behavior, 18, 237-244.

Lhermitte, F., Pillon, B., \& Serdaru, M. (1986). Human autonomy and the frontal lobes: Part 1. Imitation and utilization behavior: $\mathrm{A}$ neuropsychological study of 75 patients. Annals of Neurology, 19, 326-334

Logue, V., Durward, M., Pratt, R. T. C., Piercy, M., \& Nixon, W. L. (1968). The quality of survival after an anterior cerebral aneurysm. British Journal of Psychiatry, 114, 137-160.

Lucey, J. V., Costa, D. C., Blanes, T., Busatto, G. F., Pilowsky, L. S., Takei, N., Marks, I. M., Ell, P. J., \& Kerwin, R. W. (1995). Regional cerebral blood flow in obsessive-compulsive disorder patients at rest. British Journal of Psychiatry, 167, 629-634.

Luxenderg, J. S., Swedo, S. E., Flament, M. F., Friedland, R. P., RAPOPORT, J., \& RAPOPORT, S. I. (1988). Neuroanatomical abnormalities in obsessive-compulsive disorder detected with quantitative $\mathrm{X}$-ray computed tomography. American Journal of Psychiatry, 145, 1089-1093.

Machlin, S. R., Harris, G. J., Pearlson, G. D., Hoen-Saric, R., JefFERY, P., \& CAMARGO, E. E. (1991). Elevated medial-frontal cerebral blood flow in obsessive-compulsive patients: A SPECT study. American Journal of Psychiatry, 148, 1240-1242.

Martinot, J. L., Allilaire, J. F., Mazoyer, B. M., Hantouche, E. G., Huret, J., Legaut-Demare, F., Deslauriers, A., Hardy, P., Pappata, S., Baron, J., \& SYrota, A. (1990). Obsessive-compulsive disorder: A clinical, neuropsychological, and positron emission tomography study. Acta Psychiatrica Scandanavica, 82, 233-242.

MAXMEN, J. S., \& WARD, N. G. (1995). Essential psychopathology and its treatment. New York: Norton.

McGuire, P. K., Bench, C. J., Frith, C. D., Marks, I. M., Frackowiak, R. S. J., \& Dolan, R. J. (1994). Functional anatomy of obsessivecompulsive disorder. British Journal of Psychiatry, 164, 459-468.

MOWRER, O. H. (1960). Learning theory and behavior. New York: Wiley.

Nelson, E., EARly, T. S., \& Haller, J. W. (1993). Visual attention in obsessive-compulsive disorder. Psychiatry Research, 49, 183-196.

Nordahl, T. E., Benkelfat, C., Semple, W. E., Gross, M., King,
A. C. \& Cohen, R. M. (1989). Cerebral glucose metabolic rates in obsessive-compulsive disorder. Neuropsychopharmacology, 2, 23-28. OPPENHEIM, S., \& RoSENBERGER, J. (1991). Treatment of a case of obsessional disorder: Family systems and object relations approaches. American Journal of Family Therapy, 19, 327-333.

Pauls, D. L., Leckman, J. F., Towbin, K. E., Zahner, G. E., \& Cohen, D. J. (1986). A possible genetic relationship exists between Tourette's syndrome and obsessive-compulsive disorder. Psychopharmacology Bulletin, 22, 730-733.

Perani, D., Colombo, C., Bressi, S., Bonfanti, A., Grassi, F., Scarone, S., Bellodi, L., Smeraldi, E., \& Fazio, F. (1995). [ $\left.{ }^{18} \mathrm{~F}\right]$ FDG PET study in obsessive-compulsive disorder: A clinical/metabolic correlation study after treatment. British Journal of Psychiatry, 166, 244-250.

Perse, T. L., Greist, J. H., Jefferson, J. W., Rosenfeld, R., \& Dar, R. (1987). Flouvoxamine treatment of obsessive-compulsive disorder. American Journal of Psychiatry, 144, 1543-1548.

Pitman, R. K. (1987). A cybernetic model of obsessive-compulsive psychopathology. Comprehensive Psychiatry, 28, 334-343.

PosNer, M. I., Nissen, M. J., \& Ogden, W. C. (1978). Attended and unattended processing modes: The role of set for spatial location. In H. L. Pick, Jr., \& I. J. Saltzman (Eds.), Modes of perceiving and processing information (pp. 137-157). Hillsdale, NJ: Erlbaum.

RAPOPORT, J. L. (1991). Recent advances in obsessive-compulsive disorder. Neuropsychopharmacology, 5, 1-10.

Rasmussen, S. A., \& Tsuang, M. T. (1986). Clinical characteristic and family history in DSM-III obsessive-compulsive disorder. American Journal of Psychiatry, 143, 317-322.

Rauch, S. L., Jenike, M. A., Alpert, N. M., Baer, L., Breiter, H. C. R., Savage, C. R., \& Fischman, A. J. (1994). Regional cerebral blood flow measured during symptom provocation in obsessivecompulsive disorder using oxygen 15-labeled carbon dioxide and positron emission tomography. Archives of General Psychiatry, 51, $62-70$.

Robins, L. N., Helzer, J. E., Weissman, M. M., Orvaschel, H., GruenBERG, E., BURKE, J. D., \& REGiER, D. A. (1984). Lifetime prevalence of specific psychiatric disorders in three sites. Archives of General Psychiatry, 41, 949-958.

Robinson, D., Wu, H., Munne, R. A., Ashtari, M., Alvir, J. M.-J., Lerner, G., Koreen, A., Cole, K., \& Bogerts, B. (1995). Reduced caudate nucleus volume in obsessive-compulsive disorder. Archives of General Psychiatry, 52, 393-398.

RoLIs, E. T. (1985). Connections, functions and dysfunctions of limbic structures, the prefrontal cortex and hypothalamus. In M. Swash \& C. Kennard (Eds.), Scientific basis of clinical neurology (pp. 201 213). New York: Churchill Livingstone.

Rubin, R. T., Ananth, J., Villanueva-Meyer, J., Trajmar, P. G., \& MENA, I. (1995). Regional ${ }^{133}$ xenon cerebral blood flow and cerebral $99 \mathrm{~m}$ Tc-HMPAO uptake in patients with obsessive compulsive disorder before and during treatment. Biological Psychiatry, 38, 429-437.

Rubin, R. T., Villanueva-Meyer, J., Ananth, J., Trajmar, P. G., \& Mena, I. (1992). Regional xenon 133 cerebral blood flow and cerebral technetium $99 \mathrm{~m}$ HMPAO uptake in unmedicated patients with obsessive compulsive disorder and matched normal control subjects: Determination by high-resolution single-photon emission computed tomography. Archives of General Psychiatry, 49, 695-702.

SALKoVSKIS, P. M., \& WARWICK, H. M. C. (1985). Cognitive therapy of obsessive-compulsive disorder: Treating treatment failures. Behavioral Psychotherapy, 13, 243-255.

Scarone, S., Colombo, C., Livian, S., Abbruzzese, M., Ronchi, P., Locatelli, M., Scotti, G., \& Smeraldi, E. (1992). Increased right caudate nucleus size in obsessive-compulsive disorder: Detection with magnetic resonance imaging. Psychiatry Research: Neuroimaging, 45, 115-121.

Schwartz, J. M., Stoessel, P. W., Baxter, L. R., Martin, K. M., \& PHELPS, M. E. (1996). Systematic changes in cerebral glucose metabolic rate after successful behavior modification treatment of obsessivecompulsive disorder. Archives of General Psychiatry, 53, 109-113.

Stanley, M. A., \& Prather, R. C. (1993). Obsessive-compulsive disorder. In A. S. Bellack \& M. Hersen (Eds.), Psychopathology in adulthood (pp. 164-178). Boston: Allyn \& Bacon.

Stein, D. J., \& Holl ander, E. (1992). Cognitive science and obsessive- 
compulsive disorder. In D. J. Stein \& J. E. Young (Eds.), Cognitive science and clinical disorders (pp. 235-246). San Diego: Academic Press.

Stein, D. J., \& Hollander, E. (1994). A neural network approach to obsessive-compulsive disorder. Journal of Mind \& Behavior, 15 223-237.

Stein, D. J. Hollander, E., Chan, S., Decaria, C. M., Hilal, S., Liebowitz, M. R., \& KLEIN, D. F. (1993). Computed tomography and neurological soft signs in obsessive compulsive disorder. Psy. chiatry Research, 50, 143-150.

Swedo, S. E., Pietrini, P., Leonard, H. L., Schapiro, M. B., Rettew, D. C., Goldberger, E. L., RAPOPORT, S. I., Rapoport, J. L., \& GradY, C. L. (1992). Cerebral glucose metabolism in childhoodonset obsessive-compulsive disorder: Revisualization during pharmacotherapy. Archives of General Psychiatry, 49, 690-694.

Swedo, S. E., Rapoport, J. L., Cheslow, D. L., Leonard, H. L. AYouB, E. M., Hosier, D. M., \& WALD, E. R. (1989). High prevalence of obsessive-compulsive symptoms in patients with Sydenham's chorea. American Journal of Psychiatry, 146, 246-249.

Swedo, S. E., Schapiro, M. B., Grady, C. L., Cheslow, D. L. Leonard, H. L., Kumar, A., Friedland, R., Rapoport, S. I., \& RAPOPORT, J. (1989). Cerebral glucose metabolism in childhoodonset obsessive-compulsive disorder. Archives of General Psychiatry, 46, 518-523

Sweeney, J. A., Palumbo, D. R., Halper, J. P., \& Shear, M. K. (1992), Pursuit eye movement dysfunction in obsessive-compulsive disorder. Psychiatry Research, 42, 1-11.

Swerdlow, N. R., Benbow, C. H., Zisook, S., Geyer, M. A., \& BrafF, D. L. (1993). A preliminary assessment of sensorimotor gating in pa- tients with obsessive-compulsive disorder. Biological Psychiatry, 33, 298-301.

Tien, A. Y., Pearlson, G. D., Machlin, S. R., Bylsma, F. W., \& HOEHN-SARIC, R. (1992). Oculomotor performance in obsessivecompulsive disorder. American Journal of Psychiatry, 149, 641-646. Towey, J. P., Tenke, C. E., Bruder, G. E., Leite, P., Friedman, D., Liebowitz, M., \& Hollander, E. (1994). Brain event-related potential correlates of overfocused attention in obsessive-compulsive disorder. Psychophysiology, 31, 535-543.

TriVEDI, M. H. (1996). Functional neuroanatomy of obsessivecompulsive disorder. Journal of Clinical Psychiatry, 57(Suppl.), 26-35.

TuRnER, S. M., \& MiChelson, L. (1984). Obsessive-compulsive disorder. In S. M. Turner (Ed.), Behavioral theories and treatment of anxiety (pp. 239-277). New York: Plenum.

WARREN, R., \& Zgourides, G. D. (1991). Anxiety disorders: A rationalemotive perspective. New York: Pergamon.

Williams, K. E., \& Chambless, D. L. (1994). Behavioral therapies. In B. B. Wolman \& G. Strucker (Eds.), Anxiety and related disorders: $A$ handbook (pp. 358-375). New York: Wiley.

Zohar, J., INSEl, T. R., Berman, K. F., FoA, E. B., Hill, J. L., \& WeINBERGER, D. R. (1989). Anxiety and cerebral blood flow during behavioral challenge: Dissociation of central from peripheral and subjective measures. Archives of General Psychiatry, 46, 505-510.

(Manuscript received August 16, 1996; revision accepted for publication September 26, 1997.) 